\title{
Session-by-Session Improvements in Subjective Wellbeing and Satisfaction with Life Prospectively Predicts Session-by-Session Improvements in Bulimia Nervosa Symptoms.
}

\section{Laura E Boyajian ( $\square$ leb338@drexel.edu )}

Drexel University https://orcid.org/0000-0003-2360-9663

\section{Paakhi Srivastava}

Drexel University

\author{
Alexandra L. Pitts \\ Drexel University
}

Adrienne S. Juarascio

Drexel University

\section{Research Article}

Keywords: bulimia nervosa, eating disorder symptomatology, satisfaction with life, subjective wellbeing, behavioral treatments

Posted Date: September 7th, 2021

DOI: https://doi.org/10.21203/rs.3.rs-788376/v1

License: (c) (i) This work is licensed under a Creative Commons Attribution 4.0 International License.

Read Full License 


\section{Abstract}

\section{Purpose}

Behavioral treatments (BT) have been credited for improving both subjective wellbeing (SWB) and satisfaction with life (SWL) among those with bulimia nervosa (BN); However, researchers have yet to examine whether the improvements in SWB and SWL during BTs occur prior to or after BN symptom reduction. The current study examines session-by-session change in SWB and SWL and BN symptoms.

\section{Methods}

The sample was comprised of 35 patients with BN-spectrum disorders. Participants received 20 sessions of BT and completed weekly pre-session surveys assessing their symptom frequency, SWB, and SWL. Data was analysed using linear mixed effect modelling.

\section{Results}

BN symptom reduction during preceding sessions prospectively predicted improvements in SWB and SWL during succeeding sessions. In addition, improvements in SWB and SWL during preceding sessions prospectively predicted reductions in BN symptoms in succeeding sessions.

\section{Conclusion}

This study provides preliminary evidence of a bidirectional relationship between SWB and SWL and BN symptoms. Future research should test whether incorporating content focused on improving SWB and SWL into BT models could optimize treatment outcomes for BN.

Level I, randomized controlled trials

\section{What Is Already Known On This Subject?}

We know that behavioral treatments are associated with improved SWB and SWL among those with EDs. These studies have used cross-sectional and pre-post data, limiting our ability to understand the mechanistic process by which these changes occur. This is the first study to examine the temporal order of change in SWB and SWL and BN symptoms utilizing session-by-session data among individuals with BN-spectrum disorders.

\section{What this study adds?}

This study provides preliminary evidence towards a bidirectional relationship between SWB, SWL, and BN symptoms. These findings suggest that SWB and SWL may be mechanism of change in reducing BN symptoms during BT and that treatment efficacy may be improved by integrating content into BTs for EDs that directly focus on improving SWB and SWL. 


\section{Introduction}

Subjective wellbeing (SWB; "a person's cognitive and affective evaluation of his or her life as a whole" [1]) and satisfaction with life (SWL; "a global assessment of a person's quality of life according to his [or her] chosen criteria" [2]), commonly improve after behavioral treatment (BT), despite that the content of most BTs primarily target symptom reduction [3,4]. For example, among those with eating disorders (EDs), a population with particularly low levels of SWB and SWL [5,6], undergoing BT is associated with improved SWL $[7,8]$. To date, it has been hypothesized that reductions in ED symptoms temporally precede and ultimately lead to the observed improvements in SWB and SWL that arise after BT for EDs [9]. For instance, if an individual with bulimia nervosa (BN) is known to value social relationships, reductions in BN symptoms throughout treatment may lead to reduced distress when eating in front of loved ones, thus potentially leading to improved SWB and SWL.

While existing research suggests reductions in ED symptoms lead to improvements in SWB and SWL, improvements in SWB and SWL may temporally precede and facilitate reductions in ED symptoms throughout BT treatment. Numerous factors in the therapeutic process may contribute to early changes in SWB and SWL, such as hope and optimism that treatment will work, a strong therapeutic alliance with a clinician, or engagement in certain treatment recommendations such as enjoyable, rewarding alternative activities to tolerate urges to engage in ED symptoms. Given both high negative affect and deficits in positive affect have been widely recognized as maintenance factors for EDs [10,11], treatments that reduce negative affect or increase positive affect have the potential to impact symptom reduction on a session-by-session basis. For example, engaging in alternative activities during one week may broadly improve SWB and SWL, by reducing negative affect and increasing the number of pleasurable experiences someone has, which in turn, could lessen the likelihood an individual engages in certain ED symptoms (e.g., binge eating) during the following week.

If improvements in SWB and SWL occur prior to improvements in ED symptoms, this may provide valuable information about how to optimize the timing and delivery of certain BT components or suggest new components to that could be added to improve treatment outcomes. For example, we may consider introducing alternative activities earlier in BTs or increase the focus on SWB and SWL by drawing from existing treatments for depression (e.g., behavioral activation [12]) or third-wave cognitive behavioral treatments (CBTs) such as acceptance and commitment treatment (ACT), which focuses more directly on clarifying values and encourages engagement in values-consistent activities [13]. Alternatively, if symptom reduction were to temporally precede improvements in SWB and SWL, we may wish to retain a strong focus on earlier symptom reduction in BT via the early introduction of regular eating and reducing dietary restraint, both of which strongly predict the cessation of other ED symptoms (e.g., binge eating) $[14,15]$. Examining the temporal order of change in SWB and SWL and ED symptoms on a session-bysession basis would provide greater insight on the mechanistic process by which these improvements occur throughout treatment. 
To the best of our knowledge, no studies have assessed whether improvements in SWB and SWL temporally precede or succeed improvements in ED symptoms over short time intervals during BTs. The current study will investigate the temporal order of change in SWB and SWL and BN symptoms utilizing session-by-session measurements of these constructs among individuals with BN-spectrum disorders participating in outpatient BT. The study has two aims. In Aim 1, we will test the hypothesis that reduction in BN symptoms (i.e., binge eating, purging, compulsive exercise, restrictive eating) from one session to the next (e.g., Session 1-Session 2 change) prospectively predicts improvement in self-reported SWB and SWL during the next between-session period (e.g., Session 2-Session 3). In Aim 2, we will test the hypothesis that improvement in self-reported SWB and SWL (e.g., Session1-Session 2) prospectively predicts reduction in BN symptoms during the next between-session period (e.g., Session 2-Session 3).

\section{Methods}

\section{Participants and procedures}

The current study was a post-hoc analysis of data collected during a pilot randomized controlled trial (PI: [blinded for review]) evaluating the feasibility, acceptability, and efficacy of a novel acceptance-based behavioral treatment (ABBT) compared to CBT for BN [16]. The sample included 42 adults with a DSM-5 Diagnosis of $\mathrm{BN}$ spectrum disorders, i.e., full threshold $\mathrm{BN}$ or subthreshold $\mathrm{BN}$ [17].

Participants were recruited from the community and completed a phone screen with a trained screener to determine initial eligibility. Eligible participants were randomized to receive 20 sessions of manualized ABBT $(N=24)$ or CBT $(N=18)$ for BN. In both treatment conditions, sessions occurred over a five-month period in three phases: eight twice-weekly sessions, seven weekly sessions, and five biweekly sessions. Treatment was delivered by Master's- or Doctoral-level clinicians receiving weekly supervision from a licensed clinical psychologist. All participants provided informed consent and the study was approved by the [blinded for review] Institutional Review Board.

Because the current study utilized weekly session questionnaire data, we removed participants $(N=7)$ who dropped out before Session 10. Therefore, the final sample included 35 participants $\left(M{ }^{2} n_{A g e}=\right.$ 30.34, Mean $_{B M I}=25.7,94.30 \%$ female, $71.4 \%$ White). Initial analyses were run to include treatment condition as a predictor and produced similar results for the study aims across treatment conditions. Accordingly, we collapsed the data across treatment conditions to increase the sample size and to generate more reliable estimates for the study aims.

\section{Measures}

Weekly BN symptoms. In weekly pre-session surveys, participants reported the frequency of binge eating (i.e., any eating episode where loss of control was experienced, regardless of the amount of food consumed), purging episodes (i.e., total number of vomiting, laxative, and diuretics misuse episodes), days of restrictive eating (i.e., restricting or dieting to control weight), and days of compulsive exercise in the past week (i.e., exercising hard to control weight). Participants were prompted to provide the 
frequencies with the statement: "Over the past week, how many times have you ..." (e.g., binged (felt out of control of your eating, and eaten far more than a person normally would at one go)).

Satisfaction with Life. Before each session, participants rated the statement, "In terms of my overall satisfaction with my life, I am..." on a Likert scale from 0 (not at all satisfied) to 7 (perfectly satisfied).

Subjective Wellbeing. Before each session, participants rated the statement, "Overall, I would rate my general sense of well-being over the past week as..." on a Likert scale from 1 (very poor) to 7 (very good).

\section{Statistical Analyses}

For aims 1 and 2, we employed linear mixed effects modeling to analyze change in SWB, SWL, and BN symptoms over time [18]. The SWB, SWL, and BN symptoms ratings were lagged. For aim 1, separate models were conducted that simultaneously included time-lagged main effects modeled as fixed effects for each of the predictors, including each BN symptom (i.e., frequency of binge eating and purging and days of restrictive eating and compulsive exercise) and the outcomes (i.e., SWB and SWL). SWB and SWL ratings in the previous week, $\mathrm{BN}$ symptoms in the following week, and treatment conditions were entered as covariates. For aim 2, separate models were conducted that simultaneously included time-lagged main effects modeled as fixed effects for each predictor (i.e., SWB and SWL) and outcome including each $\mathrm{BN}$ symptom (i.e., frequency of binge eating and purging and days of restrictive eating and compulsive exercise). BN symptoms in the previous week, SWB and SWL the following week, and treatment conditions were entered as covariates. For both aims, we included fixed and random effects of time in the model to control for general effects of time (i.e., detrending [19]). To account for the dependence within the session-by-session data, a first order autoregressive (AR[1]) structure was used. An unstructured covariance structure was chosen, which allowed intercept and slopes to correlate. All statistical analyses were conducted using SPSS v.26 [20].

\section{Results}

\section{Aim 1: Reductions in BN symptoms prospectively predict improvements in SWL and SWB}

Statistical analyses are presented in Table 1. Significant effects of binge eating and purging on SWB were detected, such that reductions in binge eating and purging during previous between-session period prospectively predicted improvement in SWB during subsequent between-session period. Reductions in days of restrictive eating and compulsive exercise during previous between-session periods did not prospectively predict improvement in SWB during subsequent between-session periods.

Significant main effects of purging on SWL were also detected, such that reduction in purging during previous between-session periods prospectively predicted improvements in SWL during subsequent between-session periods. In addition, at trend level, reduction in binge eating during previous betweensession periods prospectively predicted improvement in SWL during subsequent between-session 
periods. Reduction in days of restrictive eating and compulsive exercise during previous between-session periods did not prospectively predict improvement in SWL during subsequent between-session periods.

\section{Aim 2: Improvements in SWL and SWB prospectively predict reductions in BN symptoms}

Statistical analyses are presented in Table 2. Significant main effects of SWB on binge eating and purging were detected, such that improvement in SWB during previous between-session periods prospectively predicted reduction in binge eating and purging during subsequent between-session periods. Additionally, improvement in SWB during previous between-session periods prospectively predicted reduction in days of compulsive exercise at a trend level during subsequent between-session periods. Improvement in SWB during previous between-session periods did not prospectively predict reductions in days of restrictive eating during subsequent between-session periods.

Significant main effects of SWL on binge eating were detected, such that improvement in SWL during previous between-session periods prospectively predicted reduction in binge eating during subsequent between-session periods. Additionally, improvement in SWL during previous between-session periods prospectively predicted reductions in days of compulsive exercise at trend level during subsequent between-session periods. Improvements in SWL during previous between-session periods did not prospectively predict reductions in purging and days of restrictive eating during subsequent betweensession periods.

\section{Discussion}

Though prior research has established an association between SWB and SWL and ED symptoms [9], the current study is the first to examine the temporal relationship between SWB and SWL and change in BN symptoms over short time intervals during BTs. Aim 1 was supported by the current findings, such that, in general, reductions in binge eating and purging in preceding sessions prospectively predicted improvements in SWB and SWL in succeeding sessions. Reductions in days of compulsive exercise and restrictive eating in preceding sessions did not predict improvements in SWB or SWL in succeeding sessions. Consistent with Aim 2, improvements in either or both SWB and SWL in preceding sessions prospectively predicted reduction in most BN symptoms (i.e., binging, purging, compulsive exercise). Of note, reductions in compulsive exercise were at trend level which may be due to low frequency of this behavior in the current sample. Improvements in SWB and SWL did not prospectively predict reductions in days of restrictive eating in succeeding sessions.

The findings from the current study suggest the temporal relationship between improvements in SWB and SWL and reduction in BN symptoms is complex and likely bidirectional in nature. Consistent with prior findings, our results indicate quality of life (QoL) related constructs (e.g., SWB and SWL) improve throughout BT for EDs [21]. Our findings also indicate SWB and SWL may be a mechanism of change in reducing $B N$ symptoms. These findings lend support to qualitative research, calling for a focus on QoL in treatment, based on the perception that QoL is influential on the onset, maintenance, and recovery from ED symptoms, among individuals reporting a history of EDs [22]. SWB and SWL could be important 
constructs to target more directly within BN treatment, seeing as reductions in binging and purging, two highly distressing BN symptoms, were associated with improved SWB and SWL in preceding sessions. Clinicians may consider more strongly encouraging the use of pleasurable alternative activities or incorporating values clarity work and value-based activities early on in treatment to facilitate reductions in BN symptoms, in addition to traditional early BT strategies, such as regular eating and reducing dietary restraint. It is possible treatments focusing on the integration of alternative activities, values, and $B T$ skills, such as ACT, could lead to improved treatment efficacy among those with BN.

\section{Limitations}

Despite the strengths of the study design (e.g., the collection of weekly session data), there are also several limitations that are important to note. First, the sample size was relatively small and predominantly White and female. Future research should look to replicate the current findings using a larger and more diverse sample. Additionally, in this sample, our findings on compulsive exercise were at trend level, so subsequent studies should further investigate the relationship between SWB and SWL and compulsive exercise. Moreover, both SWB and SWL were assessed through a single self-report item, asking participants about their overall SWB and SWL. This item was not a validated measure, making the reliability of the measure unknown, though it should be noted the items were drawn from validated measures [23]. Lastly, while the current study offers preliminary evidence towards a bidirectional relationship between SWB and SWL and BN symptoms, bidirectionality was not explicitly tested in the current study. Future studies should explicitly test bidirectionality to confirm whether this relationship exists. Researchers may consider using ecological momentary assessments (EMA) to appraise the mechanistic process and temporal order of change more accurately in SWB and SWL and BN symptoms.

\section{Declarations}

\section{Funding}

This work was supported by the National Institute of Mental Health under grant K23MH105680 to Dr. Juarascio.

\section{Conflicts of interest/Competing interest}

The authors have no conflicts of interest to disclose.

\section{Availability of data and material (data transparency)}

The data supporting the findings of this study are available from the corresponding author, Laura Boyajian, upon request.

\section{Code availability (software application or custom code)}

Not applicable 


\section{Authors' contributions}

Not applicable.

\section{Ethics approval}

This was a retrospective study of already collected data and was conducted after the completion of the clinical trials. The IRB approved the clinical trial and consent was obtained at the baseline assessment for the clinical trial.

\section{Consent to participate}

The IRB approved the clinical trial and informed consent was obtained at the baseline assessment for the clinical trial.

\section{Consent for publication}

The authors affirm that human research participants provided informed consent for publication of their data.

\section{References}

1. Diener, E., Lucas, R. E., \& Oishi, S. (2002). Subjective well-being: The science of happiness and life satisfaction. Handbook of positive psychology, 2, 63-73.

2. Shin, D. C., \& Johnson, D. M. (1978). Avowed happiness as an overall assessment of the quality of life. Social Indicators Research, 5(1-4), 475-492. https://doi.org/10.1007/bf00352944

3. Diener, E., Suh, E. M., Lucas, R. E., \& Smith, H. L. (1999). Subjective well-being: Three decades of progress. Psychological Bulletin, 125(2), 276-302. https://doi.org/10.1037/0033-2909.125.2.276

4. Jazaieri, H., Goldin, P. R., \& Gross, J. J. (2017). Treating social anxiety disorder with CBT: Impact on emotion regulation and satisfaction with life. Cognitive Therapy and Research, 41(3), 406-416. doi:10.1007/s10608-016-9762-4

5. Claydon, E. A., DeFazio, C., Lilly, C. L., \& Zullig, K. J. (2020). Life satisfaction among a clinical eating disorder population. Journal of Eating Disorders, 8(53) https://doi.org/10.1186/s40337-020-00326-z

6. de Vos, J. A., Radstaak, M., Bohlmeijer, E. T., \& Westerhof, G. J. (2018). Having an eating disorder and still being able to flourish? Examination of pathological symptoms and well-being as two continua of mental health in a clinical sample. Frontiers in Psychology, 9(2145). https://doi.org/10.3389/fpsyg.2018.02145

7. Ljotsson, B., Lundin, C., Mitsell, K., Carlbring, P., Ramklint, M., \& Ghaderi, A. (2007). Remote treatment of bulimia nervosa and binge eating disorder: a randomized trial of Internet-assisted cognitive behavioural therapy. Behaviour Resesearch and Therapy, 45(4), 649-661. https://doi.org/10.1016/j.brat.2006.06.010 
8. Schlup, B., Munsch, S., Meyer, A. H., Margraf, J., \& Wilhelm, F. H. (2009). The efficacy of a short version of a cognitive-behavioral treatment followed by booster sessions for binge eating disorder. Behaviour Resesearch and Therapy, 47(7), 628-635. https://doi.org/10.1016/j.brat.2009.04.003

9. Watson, H. J., Allen, K., Fursland, A., Byrne, S. M., \& Nathan, P. R. (2012). Does enhanced cognitive behaviour therapy for eating disorders improve quality of life? European Eating Disorder Review, 20(5), 393-399. https://doi.org/10.1002/erv.2186

10. Dingemans, A., Danner, U., \& Parks, M. (2017). Emotion regulation in binge eating disorder: A review. Nutrients, 9(11). https://doi.org/10.3390/nu9111274

11. Leehr, E. J., Krohmer, K., Schag, K., Dresler, T., Zipfel, S., \& Giel, K. E. (2015). Emotion regulation model in binge eating disorder and obesity--a systematic review. Neuroscience \& Biobehavioral Reviews, 49, 125-134. https://doi.org/10.1016/j.neubiorev.2014.12.008

12. Mazzucchelli, T. G., Kane, R. T., \& Rees, C. S. (2010). Behavioral activation interventions for wellbeing: A meta-analysis. Journal of Positive Psychology, 5(2), 105-121. https://doi.org/10.1080/17439760903569154

13. Stenhoff, A., Steadman, L., Nevitt, S., Benson, L., \& White, R. G. (2020). Acceptance and commitment therapy and subjective wellbeing: A systematic review and meta-analyses of randomised controlled trials in adults. Journal of Contextual Behavioral Science, 18, 256-272.

https://doi.org/https://doi.org/10.1016/j.jcbs.2020.08.008

14. Hildebrandt, T., Michaelides, A., Mackinnon, D., Greif, R., DeBar, L., \& Sysko, R. (2017). Randomized controlled trial comparing smartphone assisted versus traditional guided self-help for adults with binge eating. International Journal of Eating Disorders, 50(11), 1313-1322. https://doi.org/10.1002/eat.22781

15. Zendegui, E. A., West, J. A., \& Zandberg, L. J. (2014). Binge eating frequency and regular eating adherence: the role of eating pattern in cognitive behavioral guided self-help. Eating Behavior, 15(2), 241-243. https://doi.org/10.1016/j.eatbeh.2014.03.002

16. Fairburn, C. G. (2008). Cognitive behavior therapy and eating disorders. Guilford Press.

17. American Psychiatric Association. (2013). Diagnostic and statistical manual of mental disorders (5th ed.).

18. Singer, J. D., \& Willett, J. B. (2003). Applied longitudinal data analysis: Modeling change and event occurrence. Oxford University Press. https://doi.org/10.1093/acprof:oso/9780195152968.001.0001

19. Jebb, A. T., Tay, L., Wang, W., \& Huang, Q. (2015). Time series analysis for psychological research: examining and forecasting change. Frontiers in Psychology, 6(727). https://doi.org/10.3389/fpsyg.2015.00727

20. SPSS, I. (2019). IBM SPSS statistics for Windows, version 26.0. Armonk, NY: IBM Corp., 440.

21. Linardon, J., \& Brennan, L. (2017). The effects of cognitive-behavioral therapy for eating disorders on quality of life: A meta-analysis. International Jounral of Eating Disorders, 50(7), 715-730. https://doi.org/10.1002/eat.22719 
22. Mitchison, D., Dawson, L., Hand, L., Mond, J., \& Hay, P. (2016). Quality of life as a vulnerability and recovery factor in eating disorders: a community-based study. BMC Psychiatry, 16(1), 328. https://doi.org/10.1186/s12888-016-1033-0

23. Forman, E. M., Chapman, J. E., Herbert, J. D., Goetter, E. M., Yuen, E. K., \& Moitra, E. (2012). Using session-by-session measurement to compare mechanisms of action for acceptance and commitment therapy and cognitive therapy. Behavior Therapy, 43(2), 341-354. https://doi.org/10.1016/j.beth.2011.07.004

\section{Tables}

Table 1. Prospective association between BN symptom reduction and SWB and SWL improvement

\begin{tabular}{lllll} 
Previous session & Following session & $B$ & Std. Error & $p$ \\
\hline \multirow{2}{*}{ Binging } & Subjective Wellbeing & -0.02 & 0.01 & $0.01^{\star}$ \\
\cline { 2 - 5 } & Satisfaction with Life & -0.02 & 0.01 & 0.05 \\
\hline \multirow{2}{*}{ Purging } & Subjective Wellbeing & -0.02 & 0.01 & $<0.001^{\star}$ \\
\hline \multirow{2}{*}{ Compulsive Exercise } & Satisfaction with Life & -0.02 & 0.01 & $<0.001^{*}$ \\
\cline { 2 - 5 } & Subjective Wellbeing & 0.004 & 0.02 & 0.78 \\
\cline { 2 - 5 } Restrictive Eating & Satisfaction with Life & 0.01 & 0.02 & 0.54 \\
\hline & Subjective Wellbeing & -0.01 & 0.01 & 0.45 \\
\hline & Satisfaction with Life & -0.01 & 0.06 & 0.60
\end{tabular}

Note. ${ }^{*} p<.05$

Table 2. Prospective association between SWB and SWL improvement and BN symptom reduction 


\begin{tabular}{lllll} 
Previous session & Following session & $B$ & Std. Error & $p$ \\
\hline Subjective Wellbeing & Binging & -0.15 & 0.05 & $<0.01^{*}$ \\
\cline { 2 - 5 } & Purging & -0.16 & 0.06 & $0.01^{*}$ \\
\cline { 2 - 5 } & Compulsive Exercise & 0.13 & 0.07 & 0.09 \\
\cline { 2 - 5 } & Restrictive Eating & 0.03 & 0.04 & 0.43 \\
\hline Satisfaction with Life & Binging & -0.12 & 0.06 & $0.05^{\star}$ \\
\cline { 2 - 5 } & Purging & -0.09 & 0.07 & 0.17 \\
\cline { 2 - 5 } & Compulsive Exercise & 0.16 & 0.09 & 0.09 \\
\cline { 2 - 5 } & Restrictive Eating & 0.02 & 0.05 & 0.75
\end{tabular}

Note. ${ }^{*} p<.05$ 